\title{
Participação feminina como fator na decisão de compra de vestuário masculino
}

\author{
Women's participation as a determinant factor in \\ decision to purchase men's clothing
}

\author{
Alípio Ramos Veiga Neto ${ }^{1}$ \\ Eduardo Benevides de Oliveira² \\ Laís Karla da Silva Barreto ${ }^{3}$
}

\section{Resumo}

Compreender os fatores determinantes da participação feminina no modelo de decisão de compra do vestuário masculino é a que esta pesquisa se propõe. Foram identificados os antecedentes "dependência masculina", "envolvimento e influência feminina", e consequentes "decisão do homem", "percepção feminina dos atributos" e "categorias do vestuário". Utilizando-se tratamentos estatísticos multivariados, foram analisados fatores determinantes e correlações entre 35 variáveis de dados coletados em amostra de 535 mulheres. Verificou-se que o constructo "decisão do homem" aponta correlação significativa com a "participação feminina na compra de seu vestuário", confirmando-se as hipóteses iniciais. Sugerem-se pesquisas utilizando o modelo testado, analisando e comparando a perspectiva de outros gêneros.

Palavras-chave: Vestuário masculino. Participação feminina. Decisão de compra masculina.

\section{Abstract}

This study aimed to investigate the determinants of female participation in the menswear decision buying model. We identified the antecedents as "Male

1 Doutor em Psicologia pela Pontifícia Universidade Católica de Campinas. Coordenador do Mestrado em Administração da Universidade Potiguar. Pesquisas em: comportamento do consumidor, mídias sociais, marketing verde, inovação e estratégias de negócios baseadas em behaviorismo radical e abordagens da psicologia evolucionista. E-mail: alipio@veiga.net

2 Mestre em Administração de empresas pela Universidade Potiguar.

3 Doutora em Estudos da Linguagem pela Universidade Federal do Rio Grande do Norte. Professora colaboradora do Mestrado em Administração da Universidade Potiguar. E-mail: laisbarreto@unp.br 
Dependence", "Involvement" and "Female Influence" and the consequents as "Man Decision", "Female Perception of Attributes" and "Categories of Clothing". By using multivariate statistical methods, we analyzed the determinant factors and their correlations among 35 variables of collected data in a non-probabilistic sample of 535 women. A significant correlation among the construct "Man Decision" with the "Female Participation in Purchasing" of man clothing was found, confirming the initial hypothesis. We suggest new studies using the tested model to analyze and compare other gender perspectives.

Keywords: Male clothing. Female participation. Purchase decision of man.

\section{Introdução}

Nas últimas décadas, o surgimento do conceito de um novo homem, reforçado pelo trabalho dos profissionais de marketing, colocou a imagem pessoal como o centro da identidade e da posição social, destacando, dessa forma, o cuidado com a aparência para a construção de uma imagem pública que transmita sinais de sucesso pessoal e profissional. $\mathrm{O}$ ato de comprar vestuário ainda é considerado uma atividade essencialmente feminina, pois muitos dos compradores do sexo masculino declaram não gostar de fazer alguns tipos de compras, conforme salienta Underhill (2010). Como consequência, muitos consumidores do sexo masculino que são casados ou mantêm um relacionamento estável parecem direcionar a decisão de compra de seus vestuários para suas companheiras.

No Brasil, percebe-se essa tendência em pesquisas como a de Sophia Mind (2010), a qual, em estudo com aproximadamente 2 mil mulheres das cinco regiões do país, revelou que elas são responsáveis pela decisão de compra de $66 \%$ de tudo o que é consumido pelas famílias brasileiras, ou seja, $R \$ 1,3$ trilhão gastos anualmente com bens e serviços no país estão sob decisão das mulheres, valor que transforma o Brasil em um dos maiores mercados sob decisão feminina no mundo. 0 mercado de moda e vestuário mundial sinaliza um movimento crescente e consistente nos volumes comercializados, em parte pelo crescimento demográfico e aumento de renda, como também influenciado pela 
evolução dos papéis assumidos, nas últimas décadas, pelo público feminino relativos ao consumo desses produtos.

Pesquisa realizada pelo Ibope Solution sobre a influência das mulheres nas decisões de compra de produtos mostrou que o item "roupas masculinas" alcançou o maior percentual entre as diversas categorias de produtos pesquisados, atingindo o expressivo índice de $80 \%$, demonstrando que, apesar de produtos segmentados, o movimento feminino em direção ao universo masculino é maior do que o contrário. Fato comprovado na prática pela Renner, uma das maiores redes de lojas de departamento do Brasil, que desde 1992 definiu reposicionar sua marca com foco no público feminino, tendo observado grande sucesso com essa estratégia também nos setores masculinos de suas lojas, no qual $70 \%$ das gravatas vendidas são compradas por mulheres e, segundo o presidente dessa empresa, se o homem realiza a compra sozinho, no dia seguinte a mulher volta para trocar metade do que ele comprou (KOTLER; KELLER, 2006).

Justificando-se pela importância em se aprofundar os estudos dessas tendências com pesquisas empíricas, objetivou-se investigar os fatores determinantes da participação feminina no modelo de decisão de compra do vestuário masculino. Para tanto, buscou-se em uma pesquisa qualitativa descritiva, a partir de uma amostra composta por 535 mulheres, identificar os constructos (variáveis latentes) antecedentes e consequentes da participação feminina na decisão de compra do vestuário masculino; identificar indicadores (variáveis manifestas) para todos os constructos envolvidos no fenômeno estudado; e verificar as suas relações de dependência.

\section{Referencial teórico}

O processo de tomada de decisão de compra dos consumidores é influenciado por fatores internos e externos, conforme se verifica em Churchill e Peter (2000), bem como em Bian e Moutinho (2011), que consideram no processo de compra do consumidor as influências sociais e as influências situacionais. Para Blackwell, Miniard e Engel 
(2006), as variáveis que influenciam no processo de decisão de compra encontram-se divididas entre as influências ambientais, as diferenças individuais e os fatores pessoais, enquanto Schiffman e Kanuk (2000) e Solomon (2008) entendem que o indivíduo, como consumidor, sofre influências psicológicas, pessoais, sociais e culturais.

Blackwell, Miniard e Engel (2006), para representar como as pessoas resolvem seus problemas rotineiros que levam à compra e ao uso de produtos de todos os tipos, desenvolveram o Modelo Processo de Decisão do Consumidor (PDC), o qual demonstra os sete maiores estágios pelos quais os consumidores passam na tomada de decisão de consumo. Apesar de terminologias diferentes, o PDC apresenta similaridades com outros modelos explicativos do comportamento do consumidor (ASSAEL, 2004; SOLOMON, 2008), nos vários fatores que os influenciam.

No contexto do marketing, o termo "influência" está orientado para resultados e ligado tanto ao ato de influenciar ou modificar o comportamento do consumidor dirigindo sua compra, como também para o fato de os consumidores influenciarem as organizações em relação a seus produtos, preços, promoções e operações. Para Blackwell, Miniard e Engel (2006), as empresas estão cada vez mais sendo influenciadas pelas necessidades e desejos do mercado, em detrimento de sua influência sobre o consumidor. Na área de estudo do comportamento do consumidor, autores como Kardes, Cronley e Cline (2010) enfatizam ainda a importância dos efeitos situacionais presentes no local e momento em que a compra ocorre e como estes podem influenciar no comportamento de compra do indivíduo. No que diz respeito às interações sociais, Blackwell, Miniard e Engel (2006) afirmam que os seres humanos estão sujeitos a influências pessoais e de grupos que alteram as suas ações e comportamentos. Para Wells e Prensky (1996) e Shiffman e Kanuk (2000), são muitas as variáveis responsáveis por influenciar a decisão de compra dos consumidores, no entanto, podem ser classificadas em três grandes grupos: diferenças individuais, influências do ambiente e processos psicológicos. 
O processo de tomada de decisão de compra do consumidor encontra-se no centro desse modelo, ladeado pelas influências do ambiente, pelos processos psicológicos e pelas diferenças individuais, em que aparece o constructo "envolvimento" como um dos determinantes da complexidade e consequente extensão desse processo (ZAICHKOWSKY, 1985 e 1994). Para Blackwell, Miniard e Engel (2006), envolvimento é o grau de relevância pessoal percebida de um produto ou serviço num contexto especial. Esses autores defendem ainda que "o grau de envolvimento pessoal é o fator mais importante que molda o tipo de comportamento do processo decisório que será seguido" (p. 106).

Uma conceituação comumente aceita de envolvimento é a que o define como a importância ou a relevância percebida do objeto, baseada nas necessidades, valores e interesses inerentes ao sujeito. Essa observação é destacada em várias pesquisas, como nos artigos de Bloch, Sherrell e Ridgway (1986), Celsi e Olson (1988), Coulter, Price e Feick (2003), Richins e Bloch (1986), e Warrington e Shim (2000).

As razões, segundo Zaichkowsky (1994), para as diversas definições e medidas de envolvimento têm relação com as diferentes aplicações do termo, que são o envolvimento com a propaganda, o envolvimento com o produto e o envolvimento com o processo decisório, o que levaria também a formas distintas de avaliar o constructo envolvimento, respectivamente: maior processamento de informação e resposta; maior percepção de diferentes atributos do produto e maior comprometimento com a escolha da marca; e maior busca de informação com maior tempo gasto na escolha certa.

No que diz respeito ao envolvimento com o processo decisório, a relevância estaria especificamente na decisão (IM; HA, 2011): o consumidor engendraria em um processo decisório cuidadoso durante a compra, o qual não continuaria após a decisão ter sido realizada (o seu nível de envolvimento com o processo decisório cairia). Nesse sentido, o envolvimento sofre uma diferenciação ligada à sua propriedade de persistência, sendo classificado como "envolvimento situacional" (situational involvement) e "envolvimento duradouro" (enduring 
involvement). Esses dois tipos de envolvimento estariam relacionados às percepções de importância do produto, sendo o primeiro um tipo de envolvimento que ocorreria apenas em situações específicas, como a de uma compra; já o segundo seria um tipo de envolvimento que representaria uma preocupação contínua com uma categoria de produto, independentemente das influências situacionais (WARRINGTON; SHIM, 2000).

O estudo do processo de decisão de compra tem que ir além das várias influências sobre os compradores e identificar realmente quem toma a decisão de comprar. Essa identificação torna-se mais complexa quando há, no grupo em estudo, distinção entre os papéis de usuário e pagante, pois gera a necessidade de os usuários persuadirem os pagantes, confundindo assim a real autoria da decisão de compra. Seo, Hathcote e Sweaney (2001) consideram que a definição de quais produtos comprar, em qual loja comprar, como e quando os produtos são usados e quem deve comprá-los é um processo complicado que envolve uma variedade de papéis e atores. Solomon (2008) ressalta que os membros de uma família ou indivíduos em um centro de compras organizacional desempenham uma série de papéis específicos quando tomam uma decisão coletiva. Trata-se de um comportamento normal, pois os indivíduos apresentam relacionamentos personalizados, baseados em seus papéis nos grupos aos quais pertencem, exibindo comportamento característico associado a cada papel que desempenhem. As decisões de consumo familiar envolvem vários papéis que podem ser assumidos pelos cônjuges, pelas crianças ou outros membros do lar, sendo normal a existência de múltiplos papéis e múltiplos atores.

\section{Procedimentos metodológicos}

Optou-se pela aplicação de investigação quantitativa, pois o estudo implicou em medir relações entre variáveis e em que grau essas relações estão presentes (Mattar, 1999). Tratou-se de um estudo exploratório, utilizado para clarificar conceitos relacionados ao modelo teórico, e um estudo descritivo, a fim coletar e testar as hipóteses teóricas. Os dados 
foram coletados utilizando levantamento de campo com interrogação direta aos sujeitos investigados. Foi aplicado um questionário constituído por uma série de 35 perguntas de estimação ou avaliação agrupadas em quatro blocos nos quais os respondentes emitiram julgamento através de uma escala tipo Likert de cinco pontos (Malhotra, 2006).

O universo definido para este trabalho foi mulheres casadas ou mantendo união estável, maiores de 17 anos, alfabetizadas e residentes em uma capital de estado com aproximadamente 800 mil habitantes, independentemente de classe social. A amostra foi definida de forma não probabilística, formada pelo critério de acessibilidade. Foram aplicados 550 questionários, sem nenhum critério planejado de estratificação, alcançando-se um total de 535 questionários válidos (2,73\% de perda), atendendo ao que determinam Hair Jr. et al. (2005, p. 484) quando consideram "mais adequada uma proporção de 10 respondentes por parâmetro" para uma distribuição normal, devendo-se aumentar essa razão para 15 respondentes por parâmetro no caso de os dados violarem as suposições de normalidade multivariada, fornecendo dessa forma tamanho amostral suficiente para minimizar o impacto do erro de amostragem.

\subsection{Modelo teórico}

Bastos (2005) e Davidovitsch (2007) apresentam modelos teóricos com antecedentes e consequentes da participação feminina na decisão de compra do vestuário masculino. A partir desses estudos, adaptouse o modelo teórico proposto para esta investigação, que apresenta os antecedentes e consequentes do constructo "participação feminina na decisão de compra do vestuário masculino" (Figura 1), os quais foram definidos com base no modelo proposto e aplicados por esses autores, bem como a partir da contribuição de Kim, Damhorst e Lee (2002), Sullivan e Heitmeyer, (2008), bem como O'Cass (2004).

Os constructos definidos para compor o modelo utilizado e os indicadores especificados para medi-los tiveram a sua relevância e adequação testadas através de uma pesquisa prévia conduzida 
pelos autores deste trabalho, aplicado em um grupo formado por conveniência de acesso, composto por 142 mulheres casadas, matriculadas em universidades da cidade em que se realizou a pesquisa, independentemente de idade e classe social. O instrumento de coleta utilizado nesta pesquisa foi adaptado de Bastos (2005) e Davidovitsch (2007), contando com 27 questões distribuídas em três módulos, de forma aleatória. Baseado nas análises dos resultados encontrados nessa investigação prévia, foram mantidos todos os constructos (central, antecedentes e consequentes), reformuladas e reespecificadas quatro questões que apresentaram dificuldades no entendimento e/ou não mediam o constructo especificado, e acrescentado para mensuração dos constructos mais oito indicadores com a finalidade de atender ao que sugerem Hair Jr. et al. (2005), quando afirmam que deve-se especificar um mínimo de três variáveis de mensuração (indicadores) por constructo estudado.

Figura 1 - Modelo de decisão de compra de vestuário masculino: fatores determinantes da participação feminina.

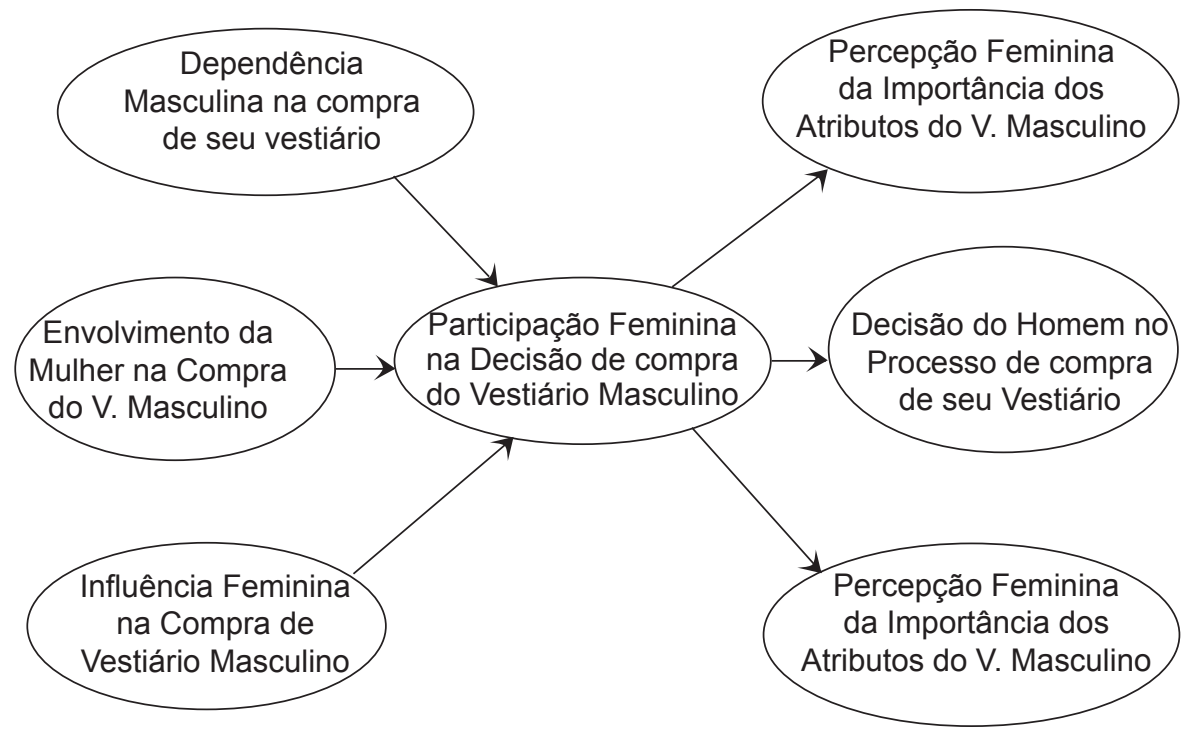

Fonte: Elaborado pelos autores. 


\section{Hipóteses}

As hipóteses foram divididas em básicas (relações diretas entre o constructo central e seus antecedentes e consequentes, envolvem o ponto central da pesquisa: $\mathrm{H} 1, \mathrm{H} 2, \mathrm{H} 3, \mathrm{H} 4, \mathrm{H} 5$ e H6) e secundárias (relação entre os diversos constructos antecedentes e consequentes e o constructo consequente "decisão do homem no processo de compra de seu vestuário" - não envolvendo, portanto, o constructo central: $\mathrm{H} 7$, H8, H9, H10 e H11), de acordo com sua relevância para os objetivos definidos para a pesquisa (Lakatos e Marconi, 2010).

A participação feminina na decisão de compra do vestuário masculino, em consonância com Bastos (2005), Blackwell, Miniard e Engel (2006), Davidovitsch (2007) e Davis e Rigaux (1974), tem como principais antecedentes a dependência masculina, o envolvimento e a influência feminina, esperando-se, dessa forma, um efeito positivo (mesmo sentido) no constructo central:

H1: Quanto maior a dependência masculina na compra de seu vestuário, maior será a participação feminina na decisão de compra do vestuário masculino.

H2: Quanto maior o envolvimento da mulher, maior será a participação feminina na decisão de compra do vestuário masculino.

H3: Quanto maior a influência feminina na compra do vestuário masculino, maior será a participação feminina na decisão de compra do vestuário masculino.

Fundamentado em Churchill e Peter (2000), Schiffman e Kanuk (2000), Sheth, Mittal e Newman (2001), Kim, Damhorst e Lee (2002), Assael (2004), Solomon (2008), Sullivan e Heitmeyer, (2008) e Kardes, Cronley e Cline, (2010) considerou-se que o constructo "participação feminina na decisão de compra do vestuário masculino" e seus antecedentes, também individualmente, apresassem relação negativa (sentido inverso) com a decisão do homem no processo de compra de seu vestuário: 
H5: Quanto maior a participação feminina na compra do vestuário masculino, menor será a decisão do homem no processo de compra do seu vestuário

H7: Quanto maior a dependência masculina na compra de seu vestuário, menor será a decisão do homem no processo de compra do seu vestuário.

H8: Quanto maior a influência feminina, menor será a decisão do homem no processo de compra do seu vestuário.

H9: Quanto maior o envolvimento da mulher, menor será a decisão do homem no processo de compra do seu vestuário.

Por fim, com relação à percepção feminina da importância dos atributos e categorias do vestuário masculino, considerou-se, em consonância com o que preconizam Blackwell, Miniard e Engel (2006), Davidovitsch (2007), O'Cass (2004) e Solomon (2008), que houvesse relação positiva (mesmo sentido) da participação feminina na decisão de compra do vestuário masculino sobre esses constructos, e que a relação direta (sem participação do constructo central) desses dois constructos com a decisão do homem no processo de decisão de compra do seu vestuário seja nula (inexistente):

H4: Quanto maior a participação feminina na compra do vestuário masculino, maior será a percepção feminina da importância dos diferentes atributos do vestuário masculino.

H6: Quanto maior a participação feminina na compra do vestuário masculino, maior será a percepção feminina da importância das diferentes categorias do vestuário masculino.

H10: A percepção feminina da importância dos atributos do vestuário masculino não interfere diretamente na decisão do homem no processo de compra do seu vestuário.

H11: A percepção feminina da importância das categorias do vestuário masculino não interfere diretamente na decisão do homem no processo de compra do seu vestuário. 
Esta pesquisa teve como instrumento de coleta de dados um questionário com perguntas de estimação ou avaliação, aplicado através de abordagem direta. Foi realizado um pré-teste em uma amostra definida pelo critério de acessibilidade, composta por 142 universitárias casadas, para verificação da sua real aderência aos objetivos propostos, o qual resultou em algumas modificações a fim de tornar as questões mais claras e o questionário mais objetivo.

O questionário definitivo aplicado foi estruturado em cinco blocos, sendo os quatro primeiros blocos formados por questões destinadas a mensurar os constructos pesquisados (35 questões), enquanto o quinto bloco (4 questões) destinou-se à coleta de dados acerca do perfil sociodemográfico dos respondentes com a finalidade de melhor caracterizar os sujeitos da pesquisa (Quadro 1).

No quinto bloco, perfil sociodemográfico, foram utilizadas escalas de mensuração do tipo nominal (SD4 - ocupação) e ordinal (SD1 - idade, SD2 - escolaridade e SD3 - renda). Com relação aos locais onde foram aplicados os questionários válidos, 239 foram aplicados em Instituições de Ensino Superior (44,67\%), 153 em shopping centers estruturados (28,60\%), 69 em áreas de comércio popular (12,90\%), 55 em um parque ecológico $(10,28 \%)$ e 19 em locais públicos diversos $(3,55 \%)$.

Quadro 1 - Constructos e variáveis manifestas.

\begin{tabular}{|l|l|}
\hline Questionário & Variáveis Manifestas \\
\hline $\begin{array}{l}\text { Constructo “Participação Feminina na Decisão de Compra do Vestuário } \\
\text { Masculino” - PF }\end{array}$ & $\begin{array}{l}\text { PF1 - Mulher acompanhar o homem na compra de } \\
\text { suas roupas }\end{array}$ \\
\hline Bloco I / Questão 1 & $\begin{array}{l}\text { PF2 - Mulher ter prazer em comprar com o homem } \\
\text { suas roupas }\end{array}$ \\
\hdashline Bloco I / Questão 2 & PF3 - Mulher avaliar com o homem esse tipo de \\
Bloco I / Questão 3 & compra \\
\hline Constructo "Dependência Masculina na Compra de seu Vestuário” - DM \\
\hdashline Bloco I / Questão 8 & $\begin{array}{l}\text { DM1 - Homem gostar de ser incentivado a vestir-se } \\
\text { bem }\end{array}$ \\
\hline
\end{tabular}




\begin{tabular}{|c|c|}
\hline Bloco I / Questão 9 & DM2 - Homem pedir recomendações sobre vestir \\
\hline Bloco I / Questão 10 & $\begin{array}{l}\text { DM3 - Homem aprovar o gosto da mulher para suas } \\
\text { roupas }\end{array}$ \\
\hline \multicolumn{2}{|c|}{$\begin{array}{l}\text { Constructo "Envolvimento da Mulher na Compra do Vestuário Masculino" - } \\
\text { EM }\end{array}$} \\
\hline Bloco II / Questão 1 & $\begin{array}{l}\text { EM1 - Mulher gostar de comprar roupa para o } \\
\text { homem }\end{array}$ \\
\hline Bloco II / Questão 2 & $\begin{array}{l}\text { EM2 - Mulher analisar detalhes na compra das } \\
\text { roupas para o homem }\end{array}$ \\
\hline Bloco II / Questão 3 & $\begin{array}{l}\text { EM3 - Mulher acertar na compra de roupa para o } \\
\text { homem }\end{array}$ \\
\hline Bloco II / Questão 4 & $\begin{array}{l}\text { EM4 - Mulher considerar importante esse tipo de } \\
\text { compra }\end{array}$ \\
\hline \multicolumn{2}{|c|}{ Constructo "Influência Feminina na Compra do Vestuário Masculino" - IF } \\
\hline Bloco I / Questão 4 & $\begin{array}{l}\text { IF1 - Mulher ser responsável pelas compras das } \\
\text { roupas do homem }\end{array}$ \\
\hline Bloco I / Questão 5 & IF2 - Mulher orientar o vestir do homem \\
\hline Bloco I / Questão 6 & $\begin{array}{l}\text { IF3 - Mulher gostar de surpreender no estilo da } \\
\text { roupa do homem }\end{array}$ \\
\hline Bloco I / Questão 7 & $\begin{array}{l}\text { IF4 - Mulher preferir presentear o homem com } \\
\text { roupas }\end{array}$ \\
\hline \multicolumn{2}{|c|}{$\begin{array}{l}\text { Constructo "Percepção Feminina da Importância dos Diferentes Atributos } \\
\text { do Vestuário Masculino" - IA }\end{array}$} \\
\hline Bloco III / Questão 1 & IA1 - Importância do conforto \\
\hline Bloco III / Questão 2 & IA2 - Importância do bom corte (modelagem) \\
\hline Bloco III / Questão 3 & IA3 - Importância do estilo \\
\hline Bloco III / Questão 4 & IA4 - Importância da marca (grife) \\
\hline Bloco III / Questão 5 & IA5 - Importância da relação entre qualidade e preço \\
\hline Bloco III / Questão 6 & IA6 - Importância da atualidade do modelo (moda) \\
\hline Bloco III / Questão 7 & IA7 - Importância da funcionalidade \\
\hline Bloco III / Questão 8 & IA8 - Importância do preço \\
\hline Bloco III / Questão 9 & IA9 - Importância da qualidade \\
\hline Bloco III / Questão 10 & IA10- Importância da durabilidade \\
\hline \multicolumn{2}{|c|}{$\begin{array}{l}\text { Constructo "Decisão do Homem no Processo de Compra de seu Vestuário" } \\
\text { - DH }\end{array}$} \\
\hline Bloco II / Questão 5 & $\begin{array}{l}\text { DH1 - Homem ter a iniciativa de "ir às compras" de } \\
\text { suas roupas }\end{array}$ \\
\hline Bloco II / Questão 6 & DH2 - Homem definir a forma de pagamento \\
\hline
\end{tabular}




\begin{tabular}{|l|l|}
\hline Bloco II / Questão 7 & $\begin{array}{l}\text { DH3 - Homem não partilhar com mulher a decisão } \\
\text { de compra }\end{array}$ \\
\hline Bloco II / Questão 8 & DH4 - Homem decidir que roupa comprar \\
\hline $\begin{array}{l}\text { Percepção Feminina da Importância das Diferentes Categorias do Vestuário } \\
\text { Masculino - IC }\end{array}$ & IC1 - Importância roupas íntimas (underwear) \\
\hline Bloco IV / Questão 1 & IC2 - Importância roupas de praia (banho) \\
\hline Bloco IV / Questão 2 & IC3 - Importância roupas para lazer \\
\hline Bloco IV / Questão 3 & IC4 - Importância roupas para trabalho (dia a dia) \\
\hline Bloco IV / Questão 4 & IC5 - Importância roupas social informal (casual) \\
\hline Bloco IV / Questão 5 & IC6 - Importância roupas social formal \\
\hline Bloco IV I Questão 6 & IC7 - Importância roupas de dormir \\
\hline Bloco IV / Questão 7 &
\end{tabular}

Fonte: Elaborado pelos autores.

No processamento dos dados coletados, foram empregadas técnicas estatísticas de natureza multivariada, destacando-se as estatísticas descritivas associadas à tendência central e à dispersão da amostra (média e desvio padrão), bem como o teste de KaiserMeyer-Olkin de Medida de Adequação da Amostra (KMO), a análise de confiabilidade (Alpha de Cronbach) e outros métodos estatísticos multivariados associados à Análise Fatorial, de acordo com Hair Jr. et al. (2005).

\section{Resultados}

\subsection{Análise descritiva: variáveis manifestas e constructos}

Apesar das médias e desvios padrão de todas as variáveis manifestas do constructo "participação feminina" alcançarem valores próximos, a variável PF3 (mulher avaliar com o homem esse tipo de compra) alcançou posição de destaque, obtendo o maior escore de grau 5 (344 ou $64,30 \%$ ), conforme pode ser observado na Tabela 1. 
Tabela 1 - Estatística descritiva do constructo "participação feminina".

\begin{tabular}{|c|c|c|c|c|c|c|c|c|c|c|c|c|c|}
\hline \multicolumn{14}{|c|}{ Participação Feminina na Compra do Vestuário Masculino } \\
\hline \multirow{2}{*}{$\begin{array}{l}\text { Variável } \\
\text { Manifesta }\end{array}$} & \multirow{2}{*}{$\mathbf{N}$} & \multicolumn{2}{|c|}{ Não nunca } & \multicolumn{2}{|c|}{ Raramente } & \multicolumn{2}{|c|}{ Às vezes } & \multicolumn{2}{|c|}{$\begin{array}{c}\text { Quase } \\
\text { sempre }\end{array}$} & \multicolumn{2}{|c|}{ Sim, sempre } & \multirow{2}{*}{ Média } & \multirow{2}{*}{$\begin{array}{l}\text { Desvio } \\
\text { Padrão }\end{array}$} \\
\hline & & $\mathbf{F}$ & $\%$ & $\mathbf{F}$ & $\%$ & $\mathbf{F}$ & $\%$ & $\mathbf{F}$ & $\%$ & $\mathbf{F}$ & $\%$ & & \\
\hline PF1 & 535 & 96 & 17,94 & 51 & 9,53 & 59 & 11,03 & 55 & 10,28 & 274 & 51,21 & 3,67 & 1,586 \\
\hline PF2 & 535 & 64 & 11,96 & 27 & 5,05 & 68 & 12,71 & 95 & 17,76 & 281 & 52,52 & 3,94 & 1,389 \\
\hline PF3 & 535 & 95 & 17,76 & 21 & 3,93 & 38 & 7,10 & 37 & 6,92 & 344 & 64,30 & 3,96 & 1,572 \\
\hline
\end{tabular}

Fonte: Dados da pesquisa.

$\mathrm{Na}$ análise descritiva do constructo "dependência masculina", nota-se equilíbrio de comportamento nas variáveis DM1 e DM3, com médias e desvios padrão semelhantes. A variável DM2 (homem pedir recomendações sobre vestir) alcançou média mais baixa que as demais e maior desvio padrão, apresentando ainda uma elevada concentração de grau 1 (187 ou 34,95\%), ao contrário das variáveis DM1 e DM2, que obtiveram mais da metade de suas respostas com grau 5 , alcançando $52,52 \%$ e 53,64\%, respectivamente (Tabela 2 ).

Tabela 2 - Estatística descritiva do constructo "dependência masculina".

\begin{tabular}{|c|c|c|c|c|c|c|c|c|c|c|c|c|c|}
\hline \multicolumn{14}{|c|}{ Dependência Masculina na Compra de seu Vestuário } \\
\hline \multirow{2}{*}{$\begin{array}{l}\text { Variável } \\
\text { Manifesta }\end{array}$} & \multirow{2}{*}{$\mathbf{N}$} & \multicolumn{2}{|c|}{ Não nunca } & \multicolumn{2}{|c|}{ Raramente } & \multicolumn{2}{|c|}{ Às vezes } & \multicolumn{2}{|c|}{$\begin{array}{l}\text { Quase } \\
\text { sempre }\end{array}$} & \multicolumn{2}{|c|}{ Sim, sempre } & \multirow{2}{*}{ Média } & \multirow{2}{*}{$\begin{array}{l}\text { Desvio } \\
\text { Padrão }\end{array}$} \\
\hline & & $\mathbf{F}$ & $\%$ & $\mathbf{F}$ & $\%$ & $\mathbf{F}$ & $\%$ & $\mathbf{F}$ & $\%$ & $\mathrm{~F}$ & $\%$ & & \\
\hline DM1 & 535 & 42 & 7,85 & 52 & 9,72 & 82 & 15,33 & 78 & 14,58 & 281 & 52,52 & 3,94 & 1,332 \\
\hline DM2 & 535 & 187 & 34,95 & 24 & 4,49 & 79 & 14,77 & 70 & 13,08 & 175 & 32,71 & 3,04 & 1,699 \\
\hline DM3 & 535 & 28 & 5,23 & 50 & 9,35 & 111 & 20,75 & 59 & 11,03 & 287 & 53,64 & 3,99 & 1,262 \\
\hline
\end{tabular}

Fonte: Dados da pesquisa.

Dentre as variáveis manifestas do constructo "envolvimento da mulher" (Tabela 3), a variável EM4 (mulher considerar importante esse tipo de compra) obteve o menor desvio padrão, a maior frequência de escores 5 (380 ou 71,03\%) e a maior média de todas as variáveis pesquisadas, independentemente de constructo, demonstrando a 
elevada importância que as mulheres pesquisadas dispensam ao escolher que roupa comprar para seus maridos/companheiros. Cabe frisar que a variável EM3 (mulher acertar na compra de RM) foi apurada com escala invertida $(5=1,4=2,3=3,2=4$ e $1=5)$.

Tabela 3 - Estatística descritiva do constructo "envolvimento da mulher".

\begin{tabular}{|c|c|c|c|c|c|c|c|c|c|c|c|c|c|}
\hline \multicolumn{14}{|c|}{ Envolvimento da Mulher na Compra do Vestuário Masculino } \\
\hline \multirow{2}{*}{$\begin{array}{l}\text { Variável } \\
\text { Manifesta }\end{array}$} & \multirow{2}{*}{$\mathbf{N}$} & \multicolumn{2}{|c|}{ Não nunca } & \multicolumn{2}{|c|}{ Raramente } & \multicolumn{2}{|c|}{ Às vezes } & \multicolumn{2}{|c|}{$\begin{array}{c}\text { Quase } \\
\text { sempre }\end{array}$} & \multicolumn{2}{|c|}{ Sim, sempre } & \multirow{2}{*}{ Média } & \multirow{2}{*}{$\begin{array}{l}\text { Desvio } \\
\text { Padrão }\end{array}$} \\
\hline & & $\mathbf{F}$ & $\%$ & $\mathbf{F}$ & $\%$ & $\mathbf{F}$ & $\%$ & $\mathbf{F}$ & $\%$ & $\mathbf{F}$ & $\%$ & & \\
\hline EM1 & 535 & 53 & 9,91 & 24 & 4,49 & 100 & 18,69 & 91 & 17,01 & 267 & 49,91 & 3,93 & 1,325 \\
\hline EM2 & 535 & 17 & 3,18 & 23 & 4,30 & 82 & 15,33 & 85 & 15,89 & 328 & 61,31 & 4,28 & 1,072 \\
\hline EM3* & 535 & 2 & 0,37 & 16 & 2,99 & 110 & 20,56 & 162 & 30,28 & 245 & 45,79 & 4,18 & 0,866 \\
\hline EM4 & 535 & 1 & 0,19 & 2 & 0,37 & 38 & 7,10 & 114 & 21,31 & 380 & 71,03 & 4,63 & 0,649 \\
\hline
\end{tabular}

* Variável com escala invertida

Fonte: Dados da pesquisa.

A análise descritiva do constructo "influência feminina" (Tabela 4) apresentou equilíbrio entre as médias das variáveis IF2, IF3 e IF4 e um afastamento da variável IF1 (mulher ser responsável pelas compras das RM), que obteve uma considerável frequência de escores 1 (216 ou $40,37 \%$ ), sinalizando que, apesar de participar da decisão de compra do vestuário masculino, a maioria das mulheres (não nunca + raramente + às vezes $=61,49 \%$ ) não se considera responsável pelas compras das roupas de seu marido/companheiro. No que diz respeito ao desvio padrão, a variável IF3 (mulher gostar de surpreender no estilo de RM) alcançou o maior maior desvio padrão de todas as variáveis pesquisadas, demonstrando uma elevada dispersão dos resultados.

Tabela 4 - Estatística descritiva do constructo "influência feminina".

\begin{tabular}{|c|c|c|c|c|c|c|c|c|c|c|c|c|c|}
\hline \multicolumn{14}{|c|}{ Influência Feminina na Compra do Vestuário Masculino } \\
\hline \multirow{2}{*}{$\begin{array}{l}\text { Variável } \\
\text { Manifesta }\end{array}$} & \multirow{2}{*}{$\mathbf{N}$} & \multicolumn{2}{|c|}{ Não nunca } & \multicolumn{2}{|c|}{ Raramente } & \multicolumn{2}{|c|}{ Às vezes } & \multicolumn{2}{|c|}{ Quase sempre } & \multicolumn{2}{|c|}{ Sim, sempre } & \multirow{2}{*}{ Média } & \multirow{2}{*}{$\begin{array}{l}\text { Desvio } \\
\text { Padrão }\end{array}$} \\
\hline & & $\mathbf{F}$ & $\%$ & $\mathbf{F}$ & $\%$ & $\mathbf{F}$ & $\%$ & $\mathbf{F}$ & $\%$ & $\mathbf{F}$ & $\%$ & & \\
\hline IF1 & 535 & 216 & 40,37 & 23 & 4,30 & 90 & 16,82 & 53 & 9,91 & 153 & 28,60 & 2,82 & 1,695 \\
\hline IF2 & 535 & 64 & 11,96 & 12 & 2,24 & 65 & 12,15 & 123 & 22,99 & 271 & 50,65 & 3,98 & 1,341 \\
\hline IF3 & 535 & 166 & 31,03 & 13 & 2,43 & 54 & 10,09 & 14 & 2,62 & 288 & 53,83 & 3,46 & 1,800 \\
\hline IF4 & 535 & 35 & 6,54 & 45 & 8,41 & 151 & 28,22 & 118 & 22,06 & 186 & 34,77 & 3,70 & 1,212 \\
\hline
\end{tabular}

Fonte: Dados da pesquisa. 
Pode-se verificar, através da análise dos valores alcançados pelas médias das variáveis manifestas do constructo "importância dos atributos" (Tabela 5), que as mulheres dispensam pouca importância à marca (grife) e à atualidade do modelo (moda) - respectivamente, IA4 e IA6 - na escolha das roupas para o marido/companheiro, mas valorizam bastante a funcionalidade, a durabilidade e principalmente a qualidade (maior média do constructo e segunda maior média dentre todas as variáveis pesquisadas: 4,44) - respectivamente IA7, IA10 e IA9 - nesse tipo de compra. As demais variáveis demonstraram equilíbrio de comportamento no que se refere aos valores das médias e devios padrão. Convém ressaltar que esse constructo concentrou oito dos dez menores desvios padrão, demonstrando elevada concentração dos resultados relacionados à percepção feminina da importância dos diferentes atributos do vestuário masculino.

Tabela 5 - Estatística descritiva do constructo "importância dos atributos".

\begin{tabular}{l|c|cc|cc|cc|cc|cc|c|c}
\hline \multicolumn{2}{l}{ Percepção Feminina da Importância dos Diferentes Atributos do Vestuário Masculino } \\
\hline $\begin{array}{l}\text { Variável } \\
\text { Manifesta }\end{array}$ & N & \multicolumn{2}{|c|}{$\begin{array}{c}\text { Sem } \\
\text { Importância }\end{array}$} & \multicolumn{2}{|c|}{$\begin{array}{c}\text { Pouca } \\
\text { Importância }\end{array}$} & \multicolumn{2}{|c|}{$\begin{array}{c}\text { Razoável } \\
\text { Importância }\end{array}$} & $\begin{array}{c}\text { Importante } \\
\text { Importante }\end{array}$ & $\begin{array}{c}\text { Muito } \\
\text { Média }\end{array}$ & $\begin{array}{c}\text { Desvio } \\
\text { Padrão }\end{array}$ \\
\hline IA1 & 535 & 2 & 0,37 & 10 & 1,87 & 109 & 20,37 & 282 & 52,71 & 132 & 24,67 & 3,99 & 0,748 \\
IA2 & 535 & 3 & 0,56 & 26 & 4,86 & 74 & 13,83 & 347 & 64,86 & 85 & 15,89 & 3,91 & 0,731 \\
IA3 & 535 & 2 & 0,37 & 21 & 3,93 & 89 & 16,64 & 319 & 59,63 & 104 & 19,44 & 3,94 & 0,741 \\
IA4 & 535 & 33 & 6,17 & 175 & 32,71 & 166 & 31,03 & 66 & 12,34 & 95 & 17,76 & 3,03 & 1,187 \\
IA5 & 535 & 4 & 0,75 & 11 & 2,06 & 132 & 24,67 & 282 & 52,71 & 106 & 19,81 & 3,89 & 0,763 \\
IA6 & 535 & 29 & 5,42 & 138 & 25,79 & 177 & 33,08 & 97 & 18,13 & 94 & 17,57 & 3,17 & 1,155 \\
IA7 & 535 & 3 & 0,56 & 14 & 2,62 & 101 & 18,88 & 163 & 30,47 & 254 & 47,48 & 4,22 & 0,879 \\
IA8 & 535 & 5 & 0,93 & 17 & 3,18 & 98 & 18,32 & 287 & 53,64 & 128 & 23,93 & 3,96 & 0,796 \\
IA9 & 535 & 0 & 0,00 & 2 & 0,37 & 67 & 12,52 & 162 & 30,28 & 304 & 56,82 & 4,44 & 0,721 \\
IA10 & 535 & 2 & 0,37 & 8 & 1,50 & 71 & 13,27 & 175 & 32,71 & 279 & 52,15 & 4,35 & 0,792 \\
\hline
\end{tabular}

Fonte: Dados da pesquisa.

Dentre as variáveis manifestas do constructo "decisão de compra do homem", verifica-se que a DH3 (homem não partilhar com mulher a decisão de compra) obteve a menor média de todas as variáveis pesquisadas (Tabela 6), o que indica a existência de uma forte participação feminina na decisão de compra do vestuario masculino, na opinião das mulheres. Cabe frisar que as variáveis $\mathrm{DH} 1, \mathrm{DH} 2$ e DH3 foram apuradas com escala invertida $(5=1,4=2,3=3,2=4$ e $1=5)$ e as médias alcançadas por DH1 e DH4 ficaram entre as dez menores. 
Tabela 6 - Estatística descritiva do constructo "decisão de compra do homem".

\begin{tabular}{|c|c|c|c|c|c|c|c|c|c|c|c|c|c|}
\hline \multicolumn{14}{|c|}{ Decisão do Homem no Processo de Compra de seu Vestuário } \\
\hline \multirow{2}{*}{$\begin{array}{l}\text { Variável } \\
\text { Manifesta }\end{array}$} & \multirow{2}{*}{$\mathbf{N}$} & \multicolumn{2}{|c|}{ Não nunca } & \multicolumn{2}{|c|}{ Raramente } & \multicolumn{2}{|c|}{ Às vezes } & \multicolumn{2}{|c|}{$\begin{array}{l}\text { Quase } \\
\text { sempre }\end{array}$} & \multicolumn{2}{|c|}{ Sim, sempre } & \multirow{2}{*}{ Média } & \multirow{2}{*}{$\begin{array}{l}\text { Desvio } \\
\text { Padrão }\end{array}$} \\
\hline & & $\mathbf{F}$ & $\%$ & $\mathbf{F}$ & $\%$ & $\mathbf{F}$ & $\%$ & $\mathbf{F}$ & $\%$ & $\mathbf{F}$ & $\%$ & & \\
\hline $\mathrm{DH}^{*}$ * & 535 & 226 & 42,24 & 47 & 8,79 & 132 & 24,67 & 12 & 2,24 & 118 & 22,06 & 2,53 & 1,571 \\
\hline $\mathrm{DH} 2^{*}$ & 535 & 124 & 23,18 & 30 & 5,61 & 97 & 18,13 & 18 & 3,36 & 266 & 49,72 & 3,51 & 1,659 \\
\hline DH3* & 535 & 337 & 62,99 & 72 & 13,46 & 67 & 12,52 & 21 & 3,93 & 38 & 7,10 & 1,79 & 1,228 \\
\hline DH4 & 535 & 167 & 31,21 & 28 & 5,23 & 118 & 22,06 & 30 & 5,61 & 192 & 35,89 & 3,10 & 1,670 \\
\hline
\end{tabular}

* Variável com escala invertida

Fonte: Dados da pesquisa.

A análise estatística descritiva do constructo "importância das categorias" (Tabela 7) mostra que as variáveis IC2 (roupa para banho), IC6 (roupa social formal) e IC7 (roupa para dormir) foram as que obtiveram menores médias, presumindo-se que são as categorias que recebem menos atenção pelas mulheres na hora da compra de roupas masculinas. Em sentido inverso, a importância das roupas para lazer (IC3) obteve a maior média do constructo, indicando ser a categoria de roupas masculinas que recebe maior atenção por parte das mulheres.

Tabela 7 - Estatística descritiva do constructo "importância das categorias".

\begin{tabular}{|c|c|c|c|c|c|c|c|c|c|c|c|c|c|}
\hline \multicolumn{14}{|c|}{ Percepção Feminina da Importância das Diferentes Categorias do Vestuário Masculino } \\
\hline \multirow{2}{*}{$\begin{array}{l}\text { Variável } \\
\text { Manifesta }\end{array}$} & \multirow[t]{2}{*}{$\mathbf{N}$} & \multicolumn{2}{|c|}{$\begin{array}{c}\text { Sem } \\
\text { Importância }\end{array}$} & \multicolumn{2}{|c|}{$\begin{array}{c}\text { Pouca } \\
\text { Importância }\end{array}$} & \multicolumn{2}{|c|}{$\begin{array}{c}\text { Razoável } \\
\text { Importância }\end{array}$} & \multicolumn{2}{|c|}{ Importante } & \multicolumn{2}{|c|}{$\begin{array}{c}\text { Muito } \\
\text { Importante }\end{array}$} & \multirow[t]{2}{*}{ Média } & \multirow{2}{*}{$\begin{array}{l}\text { Desvio } \\
\text { Padrão }\end{array}$} \\
\hline & & $\mathbf{F}$ & $\%$ & $\mathbf{F}$ & $\%$ & $\mathbf{F}$ & $\%$ & $\mathbf{F}$ & $\%$ & $\mathbf{F}$ & $\%$ & & \\
\hline IC1 & 535 & 45 & 8,41 & 47 & 8,79 & 52 & 9,72 & 288 & 53,83 & 103 & 19,25 & 3,67 & 1,136 \\
\hline IC2 & 535 & 161 & 30,09 & 138 & 25,79 & 125 & 23,36 & 92 & 17,20 & 19 & 3,55 & 2,38 & 1,182 \\
\hline IC3 & 535 & 6 & 1,12 & 46 & 8,60 & 62 & 11,59 & 265 & 49,53 & 156 & 29,16 & 3,97 & 0,924 \\
\hline IC4 & 535 & 153 & 28,60 & 48 & 8,97 & 82 & 15,33 & 150 & 28,04 & 102 & 19,07 & 3,00 & 1,510 \\
\hline IC5 & 535 & 34 & 6,36 & 88 & 16,45 & 83 & 15,51 & 233 & 43,55 & 97 & 18,13 & 3,51 & 1,151 \\
\hline IC6 & 535 & 193 & 36,07 & 118 & 22,06 & 57 & 10,65 & 87 & 16,26 & 80 & 14,95 & 2,52 & 1,482 \\
\hline IC7 & 535 & 258 & 48,22 & 85 & 15,89 & 102 & 19,07 & 79 & 14,77 & 11 & 2,06 & 2,07 & 1,203 \\
\hline
\end{tabular}

Fonte: Dados da pesquisa.

Pode-se notar que três das quatro (75\%) variáveis especificadas para medir o constructo "envolvimento da mulher" posicionaram-se entre as dez maiores médias, tendo EM4 (mulher considerar importante este 
tipo de compra) alcançado a maior média detse estudo, demonstrando coerência com Bloch, Sherrell e Ridgway (1986), Celsi e Olson (1988), Coulter, Price e Feick (2003), Richins e Bloch (1986), Warrington e Shim (2000) e Zaichkowsky (1985), quando afirmam que o envolvimento consiste na importância ou relevância percebida do objeto (nesse caso, o vestuário masculino), baseada nas necessidades, valores e interesses inerentes ao sujeito (a mulher).

A análise descritiva dos constructos do modelo utilizado (Tabela 8) destacou a elevada média alcançada pelo constructo "envolvimento da mulher" (maior média do modelo, com grau 4,2528) e o baixo valor da média do constructo "decisão do homem" (menor média do modelo: 2,7308 ), o que evidencia, em uma análise preliminar, a força do constructo "envolvimento da mulher" como antecedente da participação feminina na compra do vestuário masculino, bem como o efeito acima da média que o contructo "participação feminina" (média: 3,8575) exerce na decisão do homem no processo de compra do seu vestuário, sinalizando a existência de uma relação negativa entre esses dois constructos, isto é, quando a participação feminina aumenta, a decisão do homem diminui, conforme sugerido pela hipótese H5.

Tabela 8 - Estatística descritiva dos constructos.

\begin{tabular}{l|c|c|c|c|c|c}
\hline CONSTRUCTOS & N & \multicolumn{1}{c}{ AMPLITUDE } & \multicolumn{1}{c}{ MíN. } & \multicolumn{1}{c}{ MÁX. } & \multicolumn{1}{c}{ MÉDIA } & \multicolumn{1}{c}{ DESVIO PADRÃO } \\
\hline PF - Participação Feminina & 535 & 4,00 & 1,00 & 5,00 & $\mathbf{3 , 8 5 7 5}$ & 1,22482 \\
DM - Dependência Masculina & 535 & 4,00 & 1,00 & 5,00 & $\mathbf{3 , 6 5 6 6}$ & 1,04747 \\
EM - Envolvimento da Mulher & 535 & 3,00 & 2,00 & 5,00 & $\mathbf{4 , 2 5 2 8}$ & 0,67596 \\
IF - Influência Feminina & 535 & 3,50 & 1,50 & 5,00 & $\mathbf{3 , 4 9 0 2}$ & 0,86075 \\
IA - Importância Atributos & 535 & 3,20 & 1,70 & 4,90 & $\mathbf{3 , 8 8 8 6}$ & 0,47127 \\
DH - Decisão do Homem & 535 & 4,00 & 1,00 & 5,00 & $\mathbf{2 , 7 3 0 8}$ & 1,04026 \\
IC - Importância Categorias & 535 & 2,86 & 1,71 & 4,57 & $\mathbf{3 , 0 1 5 8}$ & 0,54504 \\
\hline
\end{tabular}

Fonte: Dados da pesquisa.

Com a análise descritiva das variáveis manifestas e dos constructos realizada, passou-se à verificação da validade convergente dos constructos através da aplicação da técnica estatística da análise fatorial confirmatória, à análise do modelo proposto e ao teste das hipóteses formuladas para essa pesquisa. 


\subsection{Análise dos constructos: validade convergente}

Optou-se pela técnica estatística de análise fatorial confirmatória (AFC) para testar individualmente cada constructo componente do modelo proposto, com o objetivo de verificar se o conjunto de variáveis manifestas (indicadores) especificadas para cada um deles estavam medindo, de fato, o que teoricamente deveriam medir, ou seja, validação convergente (Aranha \& Zambaldi, 2008). Em seguida, para verificar as relações existentes entre os constructos participantes do modelo utilizado, foi aplicada a matriz de correlação de Pearson (Hair JR. et al., 2005). Os resultados encontrados forneceram a base empírica necessária para o confronto com as expectativas teóricas e verificação da validade das hipóteses apresentadas.

Inicialmente, foi investigada a validade convergente de todos os indicadores especificados para cada um os sete constructos definidos, através da aplicação da AFC, pois a utilização de variáveis inválidas colocaria sob suspeita quaisquer análises subsequentes e "não é apenas porque um pesquisador formula uma questão para medir alguma coisa que a questão de fato a mede, é preciso que várias propriedades da variável indicadora sejam confirmadas, antes que ela possa ser utilizada como medida válida" (Aranha \& Zambaldi, 2008, p. 108). A convergência das medidas, isto é, a existência de forte correlação entre medidas destinadas a mensurar um mesmo constructo (Gerbing \& Anderson, 1988), foi suportada basicamente pelo fato de 32 das 35 variáveis manifestas (indicadores) especificadas apresentarem coeficientes fatoriais significativos em relação aos seus respectivos constructos, sendo as três que não apresentaram significância teórica nem prática significativas excluídas do estudo (IF3, IC3 e IC7), pois essa condição não justificaria sua permanência.

\subsection{Análise do modelo proposto e confirmação de hipóteses}

Após a validação convergente de todos os constructos e a definição de quais indicadores iriam representá-los (média) na análise do modelo da participação feminina na decisão de compra do 
vestuário masculino utilizado nesta pesquisa, verificou-se a variância total explicada por esse modelo, bem como as relações existentes entre seus componentes (constructos). A variância total explicada pelo modelo proposto foi calculada através da aplicação da análise fatorial exploratória (excluídos os indicadores IF3, IC3 e IC7), atingindo para oito componentes (agrupando-se 32 indicadores validados) 55,736\% e para sete componentes (agrupando-se 31 indicadores validados), 52,370\%, demonstrando em ambas situações nível satisfatório de explicação, isto é, mais da metade da variância total foi explicada (Tabela 9), o que, segundo Aranha e Zambaldi (2008), é considerado um nível de explicação bom para pesquisas em ciências sociais. Cabe ressaltar que a matriz de componentes rotacionados gerada pelo software estatístico reagrupou os indicadores validados para este estudo em oito componentes (constructos), mas, para o oitavo componente (fora do escopo da pesquisa), foi especificado apenas o indicador "preço" (IA8), fato que guarda coerência com os baixos valores de comunalidade alcançados por esse indicador, tanto em relação aos indicadores do constructo Importância dos Atributos $(0,096)$ quanto em relação a todos os outros indicadores deste estudo $(0,072)$.

Com os resultados encontrados para variância total explicada, tornase evidente que o fenômeno estudado por essa pesquisa foi representado de forma satisfatória pelo modelo utilizado (sete constructos), o qual demonstrou aderência aos objetivos definidos, apresentando-se como um "conjunto especificado de relações de dependência que podem ser testadas empiricamente - uma operacionalização de uma teoria" (Hair Jr. et al., 2005, p.469), nesse caso da participação feminina. 
Tabela 9 - Variância total explicada: modelo proposto.

\begin{tabular}{l|c|c|c|c|c|c}
\hline \multirow{2}{*}{ Componentes } & \multicolumn{3}{|c|}{ Autovalores Iniciais } & \multicolumn{3}{c}{ Soma da Extração de Cargas Quadráticas } \\
\cline { 2 - 7 } & Total & $\%$ Variância & \% Acumulado & Total & \% Variância & \% Acumulado \\
\hline 1 & 6,113 & 19,103 & 19,103 & 6,113 & 19,103 & 19,103 \\
2 & 2,955 & 9,234 & 28,338 & 2,955 & 9,234 & 28,338 \\
3 & 1,998 & 6,243 & 34,581 & 1,998 & 6,243 & 34,581 \\
4 & 1,766 & 5,517 & 40,098 & 1,766 & 5,517 & 40,098 \\
5 & 1,408 & 4,400 & 44,498 & 1,408 & 4,400 & 44,498 \\
6 & 1,362 & 4,256 & 48,755 & 1,362 & 4,256 & 48,755 \\
7 & 1,157 & 3,615 & 52,370 & 1,157 & 3,615 & 52,370 \\
8 & 1,077 & 3,366 & 55,736 & 1,077 & 3,366 & 55,736 \\
\hline
\end{tabular}

Método de extração: Análise de componentes principais

Fonte: Dados da pesquisa.

Os constructos foram representados nessa análise pelas médias de seus indicadores validados (Tabela 10), conforme orientação dos autores acerca da necessidade de redução de itens de estudo através do uso de variáveis de substituição, no tratamento de modelos complexos (Aranha \& Zambaldi, 2008; Hair Jr. et al., 2005).

Tabela 10 - Novas médias e desvios padrão dos constructos (sem IF3/ IC3/IC7).

\begin{tabular}{l|c|c|c|c|c|c}
\hline CONSTRUCTOS & N & AMPLITUDE & MíN. & MÁX. & MÉDIA & DESVIO PADRÃO \\
\hline PF - Participação Feminina & 535 & 4,00 & 1,00 & 5,00 & $\mathbf{3 , 8 5 7 5}$ & 1,22482 \\
DM - Dependência Masculina & 535 & 4,00 & 1,00 & 5,00 & $\mathbf{3 , 6 5 6 6}$ & 1,04747 \\
EM - Envolvimento da Mulher & 535 & 3,00 & 2,00 & 5,00 & $\mathbf{4 , 2 5 2 8}$ & 0,67596 \\
IF - Influência Feminina & 535 & 4,00 & 1,00 & 5,00 & $\mathbf{3 , 5 0 1 0}$ & 0,98628 \\
IA - Importância Atributos & 535 & 3,20 & 1,70 & 4,90 & $\mathbf{3 , 8 8 8 6}$ & 0,47127 \\
DH - Decisão do Homem & 535 & 4,00 & 1,00 & 5,00 & $\mathbf{2 , 7 3 0 8}$ & 1,04026 \\
IC - Importância Categorias & 535 & 3,20 & 1,60 & 4,80 & $\mathbf{3 , 0 1 5 3}$ & 0,68673 \\
\hline
\end{tabular}

Fonte: Dados da pesquisa.

Para a verificação do grau de associação linear (correlação) entre os constructos formadores do modelo proposto, utilizou-se o coeficiente de Pearson (r), recomendado por Hair Jr. et al. (2005). Foram calculados também, de forma complementar, os coeficientes de Spearman e Kendall, testes não paramétricos, os quais indicaram os mesmos comportamentos identificados pelo coeficiente de Pearson (sentido do efeito), apenas com intensidade/magnitude diferentes, quase sempre mais baixas. 
Figura 3 - Correlações verificadas no modelo de decisão de compra de vestuário masculino.

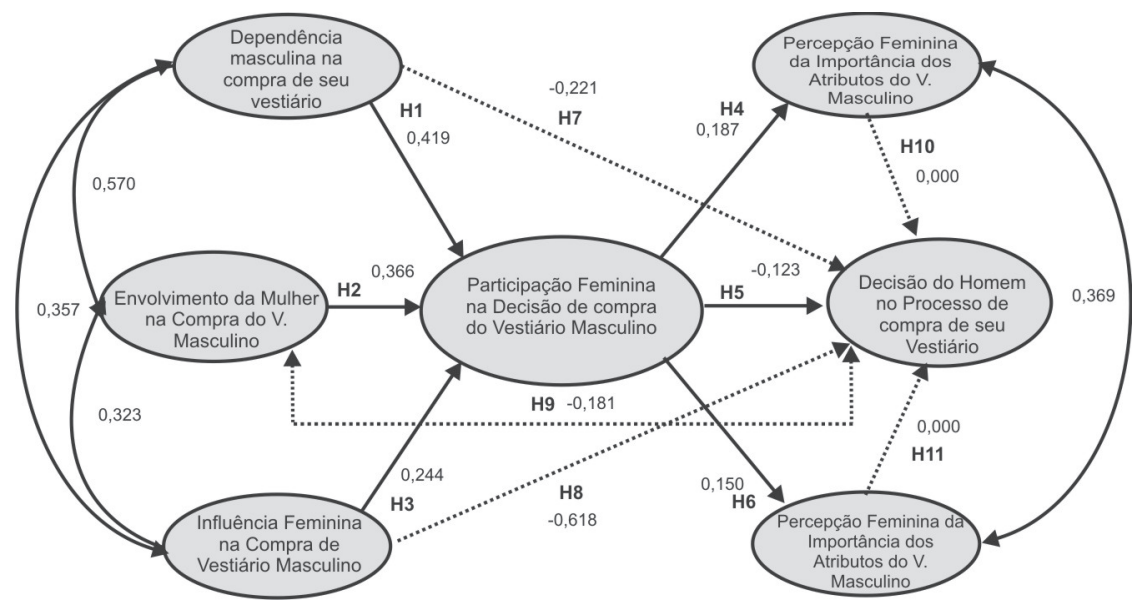

Fonte: Elaborado pelos autores.

As correlações encontradas demonstraram a conformidade do comportamento da amostra pesquisada com a expectativa teórica, que se baseou na revisão de literatura utilizada para o desenvolvimrnto do modelo da participação feminina na decisão de compra do vestuário masculino. Analisando-se as correlações apresentadas na Figura 3 , torna-se evidente que os constructos antecedentes (DM, EM e IF) relacionaram-se com o constructo central (PF) de forma significativa, apresentando todos o mesmo sentido do efeito: DM: PF = 0,419, EM: $P F=0,366$ e IF: $P F=0,244$, isto é, quando ocorre variação em qualquer um desses constructos, o reflexo no constructo central segue o mesmo sentido (sinal positivo), fato que confirma as hipóteses básicas $\mathrm{H} 1, \mathrm{H} 2$ e H3.

No que se refere às relações entre o constructo central e seus consequentes (IA, DH e IC), os valores encontrados demonstraram a existência de correlação significativa entre esses constructos e os sentidos do efeito distintos. O constructo central (PF) correlacionou-se com o constructo $\mathrm{DH}$, apresentando sentido oposto do efeito: PF:DH $=-0,123$, isto é, quando há variação no constructo central, o reflexo 
em DH segue o sentido inverso da variação (sinal negativo), enquanto em relação aos constructos IA e IC, o constructo central apresentou o mesmo sentido do efeito: PF:IA $=0,187$ e PF:IC $=0,150$, isto é, quando há variação no constructo central, o reflexo em qualquer um desses constructos segue o mesmo sentido (sinal positivo). Esses fatos confirmam as hipóteses básicas H4, H5 e H6.

As relações entre os diversos constructos antecedentes e consequentes e o constructo consequente $\mathrm{DH}$, não envolvendo o constructo central PF (hipóteses secundárias), apresentaram comportamentos distintos. Os constructos antecedentes (DM, EM e IF) relacionaram-se com o constructo dependente DM de forma significativa, apresentando todos sentido oposto do efeito: $\mathrm{DM}: \mathrm{DH}=-0,221, \mathrm{EM}: \mathrm{DH}=$ $-0,181$ e IF:DH = -0,618, isto é, as variações ocorridas em qualquer um desses constructos provocam em DH uma variação de sentido inverso (sinal negativo), resultados que confirmam as hipóteses secundárias H7, H8 e H9. Convém ressaltar que a maior correlação registrada por esse estudo foi entre os constructos "influência feminina" (IF) e "decisão do homem" (DH): 0,618. Os constructos consequentes IA e IC não apresentaram correlação com o constructo $\mathrm{DH}$ (IA:DH = 0,000 e IC:DH $=0,000)$, o que confirma o comportamento sugerido pelas hipóteses secundárias $\mathrm{H} 10$ e H11, ou seja, as variações de IA e IC não afetam de forma direta a decisão do homem no processo de compra do seu vestuário.

As correlações apresentadas entre os constructos antecedentes (DM, EM e IF) demonstraram que as relações entre os três são significantes e apresentam o mesmo sentido do efeito: DM:EM = $0,570, \mathrm{DM}: \mathrm{IF}=0,357$ e EM:IF = 0,323, isto é, as variações nesses três constructos seguem sempre um mesmo sentido (sinal positivo). Os constructos caminham juntos, o que reforça as evidências do acerto na definição desses constructos como antecedentes da participação feminina na decisão de compra do vestuário masculino. Esse mesmo comportamento repetiu-se em relação aos constructos consequentes IA e IC, que apresentaram relações significantes e mesmo sentido do efeito $(I A: I C=0,360)$. 


\section{Conclusão}

Os resultados encontrados demonstraram que, sob a ótica feminina, a mulher participa do processo de decisão de compra do vestuário masculino, influenciando a decisão do homem no processo de compra do seu vestuário (correlações identificadas). O objetivo geral foi alcançado, pois as análises realizadas no decorrer do trabalho indicaram a existência de relações entre a participação feminina e seus antecedentes e consequentes. Após a avaliação da convergência do conjunto de medidas proposto (modelo de mensuração), foi examinada a força das relações de causalidade, verificando-se as correlações entre os sete constructos do modelo estrutural através do teste paramétrico de Pearson. Todas as hipóteses formuladas a respeito das relações existentes foram apoiadas, denotando coerência entre o resultado apresentado pela amostra pesquisada (empírico) e a base teórica consultada (teórico).

Com a base teórica formada e partindo-se do pressuposto de que a operacionalização de conceitos (constructos) em variáveis empiricamente observáveis (indicadores) constitui-se em procedimento central para viabilizar a produção do conhecimento, esquematizou-se um modelo teórico (estrutural e de mensuração) com a definição de antecedentes e consequentes da participação feminina no processo de compra do vestuário masculino, o qual orientou também a formulação das hipóteses acerca desse fenômeno. A aplicação da análise fatorial possibilitou mensurar de forma adequada os constructos envolvidos, evitando-se que variáveis mal medidas (indicadores mal especificados) comprometessem a validade dos resultados, o que poderia levar a conclusões errôneas sobre o fenômeno em estudo. Apesar de os resultados da análise fatorial estarem sujeitos a dúvidas, o referencial teórico utilizado para a realização desta pesquisa, aliado às definições prévias acerca da quantidade de fatores comuns e à apresentação de uma expectativa teórica sobre qual fator deve carregar em qual variável, tornou possível minimizá-las, mantendo-se as condições necessárias para a utilização da análise fatorial confirmatória. Por tudo isso, pode- 
se concluir que os resultados deste estudo representam de forma fiel o comportamento da amostra acerca do fenômeno pesquisado.

Em termos práticos, esta pesquisa contribuiu para oaprofundamento do estudo das mudanças ocorridas nos papéis assumidos pelos gêneros masculino e feminino enquanto consumidores. Segundo Davidovitsch (2007), os papéis não são mais determinados biologicamente pelo sexo, e sim pelas experiências sociais que ensinam aos homens e às mulheres atividades diferentes enquanto consumidores. A mulher assume cada vez mais papel de relevância como consumidora, participando ativamente de todas as decisões de compra presentes no seu dia a dia profissional e/ou doméstico.

Como limitações, foi possível identificar que o critério utilizado para a formação da amostra foi o da acessibilidade (conveniência), técnica não probabilística que, por essa razão, não permite que as estimativas obtidas sejam estatisticamente projetáveis para a população, ou seja, não é recomendável fazer inferências ou projeções sobre a populaçãoalvo da qual se extraiu a amostra (MALHOTRA, 2006). Outra limitação verificada refere-se ao comportamento dos dados coletados, limitação comum às pesquisas em ciências sociais, pois, no mundo real, na maioria das vezes, os dados coletados ferem as premissas estabelecidas para a possibilidade de aplicação da análise multivariada, as quais foram minimizadas através do uso de um tamanho adequado de amostra.

Sugere-se: 1) a realização de novos estudos sobre o fenômeno abordado, mas com a utilização de amostragem aleatória simples ou até mesmo, dependendo do foco do pesquisador, uma amostragem por cluster, 2) a utilização, em estudos futuros, da modelagem de equações estruturais (SEM), técnica multivariada que combina aspectos de regressão múltipla e análise fatorial para estimar uma série de relações de dependência inter-relacionadas simultaneamente, cujos algoritmos utilizados possibilitam incorporar ao mesmo tempo os efeitos de erros de mensuração sobre os coeficientes estruturais, superando as limitações intrínsecas da técnica de análise fatorial no estudo de um modelo complexo de forma simultânea. 


\section{Referências}

ARANHA, F.; ZAMBALDI, F. Análise fatorial em administração. São Paulo: Cengage Learning, 2008.

ASSAEL, H. Consumer behavior: a strategic approach. Boston: Houghton Mifflin, 2004.

BASTOS, E. R. A influência feminina no consumo masculino de cosméticos. 2005, 157 f. Dissertação (Mestrado em Administração)Faculdades IBMEC, Rio de Janeiro, 2005.

BIAN, X.; MOUTINHO, L. The role of brand image, product involvement, and knowledge in explaining consumer purchase behaviour of counterfeits: direct and indirect effects. European Journal of Marketing, Leicestershire, v. 45, n. 1, p. 191-216, 2011.

BLACKWELL, R. D.; MINIARD, P. W.; ENGEL, J. F. Consumer behavior. Mason: Thomson South-Western, 2006.

BLOCH, P. H.; SHERRELL, D.L.; RIDGWAY, N.M. Consumer search: an extended framework. Journal of Consumer Research, Madison, v. 13, n. 1, p. 119-126, 1986.

CELSI, R. L.; OLSON, J. C. The role of involvement in attention and comprehension processes. Journal of Consumer Research, Madison, v. 15, n. 2, p. 210-224, 1988.

CHURCHILL, G. A.; PETER, J. P. Marketing: criando valor para o cliente. São Paulo: Saraiva, 2000.

COULTER, R. A.; PRICE, L. L.; FEICK, L. Rethinking the origins of involvement and brand commitment: insights from postsocialist Central Europe. Journal of Consumer Research, Madison, v. 30, n. 2, p. 151170, 2003.

DAVIDOVITSCH, L. Antecedentes e consequências dos níveis de envolvimento masculino com vestuário: uma pesquisa empírica com homens do Rio de Janeiro. 2007. 157 f, Dissertação (Mestrado em 
Administração)-Pontifícia Universidade Católica do Rio de Janeiro, Rio de Janeiro, 2007.

DAVIS, H. L.; RIGAUX, B. R. Perception of marital roles in decision process. Journal of Consumer Research, Madison, v. 1, p. 5-14, 1974.

GERBING, D. W.; ANDERSON, J. C. An updated paradigm for scale development incorporating unidimensionality and its assessement. Journal of Marketing Research, Madison, v. 15, p.186-92, 1988.

HAIR JUNIOR, J. F. et al. (2005) Análise multivariada de dados. Porto Alegre: Bookman, 2005.

IM, H.; HA, Y. The effect of perceptual fluency and enduring involvement on situational involvement in an online apparel shopping context. Journal of Fashion Marketing and Management, Manchester, v. 15, n. 3, p. 345-362, 2011.

KARDES, F. R.; CRONLEY, M. L.; CLINE, T. W. Consumer Behavior. Manson: Centage Learning, 2010.

KIM, H. S.; DAMHORST, M. L.; LEE, K. H. Apparel involvement and advertisement processing: A model". Journal of Fashion Marketing and Management, Manchester, v. 6, n. 3, p. 277-302, 2002.

KOTLER, P.; KELLER, K. L. Administração de marketing. São Paulo: Prentice Hall, 2006.

LAKATOS, E. M.; MARCONI, M. A. Fundamentos de metodologia científica. São Paulo: Atlas, 2010.

MALHOTRA, N. K. Pesquisa de marketing: uma orientação aplicada. Porto Alegre: Bookman, 2006.

MATTAR, F. N. Pesquisa de marketing: metodologia, planejamento. São Paulo: Atlas, 1999.

O'CASS, A. Fashion clothing consumption: antecedents and consequences of fashion clothing involvement. European Journal of Marketing, Leicestershire, v. 38, n. 7, p. 869-882, 2004. 
RICHINS, M. L.; BLOCH, P. H. After the new wears off: the temporal context of product involvement. Journal of Consumer Research, Madison, v. 13, n. 2, p. 280-285, 1986.

SCHIFFMAN, L. G.; KANUK, L. L. Comportamento do consumidor. São Paulo: LTC, 2000.

SEO, J. I.; HATHCOTE, J. M.; SWEANEY, A. L. Casualwear shopping behavior of college men in Georgia, USA. Journal of Marketing and Management, Westburn, v. 5, n. 3, 208-222, 2001.

SHETH, J. N.; MITTAL, B.; NEWMAN, B. I. Comportamento do cliente: indo além do comportamento do consumidor. São Paulo: Atlas, 2001.

SOLOMON, M. R. O comportamento do consumidor: comprando, possuindo e sendo. Porto Alegre: Bookman, 2008.

SOPHIA MIND. Brasileiras controlam $66 \%$ do consumo das famílias brasileiras. 2010. Disponível em: <www.sophiamind.com/pesquisas>. Acesso em: 10 jun. 2011.

SULLIVAN, P.; HEITMEYER, J. Looking at gen y shopping preferences and intentions: exploring the role of experience and apparel involvement. International Journal of Consumer Studies, Malden, v. 32, n. 3, p. 285-295, 2008.

UNDERHILL, P. What women want: the global market turns female friendly. New York: Simon and Schuster, 2010.

WARRINGTON, P.; SHIM, S. An empirical investigation of the relationship between product involvement and brand commitment. Psychology and Marketing, Malden, v. 17, n. 9, p. 761-782, 2000.

WELLS, W.; PRENSKY, D. Consumer behavior. New York: John Wiley \& Sons, 1996.

ZAICHKOWSKY, J. L. Measuring the involvement construct. Journal of Consumer Research, Madison, v. 12, n. 3, p. 341-352, 1985. 
ZAICHKOWSKY, J. L. The personal involvement inventory: reduction, revision, and application to advertising. Journal of Advertising, Philadelphia, v. 23, n. 4, p. 59-70, 1994.

Artigo recebido em: 03/06/2013 Aprovado em: 06/12/13 